\title{
A Novel Analytical Technique to Obtain Kink Solutions for Higher Order Nonlinear Fractional Evolution Equations
}

\author{
Qazi Mahmood Ul Hassan, Jamshad Ahmad, and Muhammad Shakeel \\ Department of Mathematics, Faculty of Sciences, HITEC University Taxila Cantt Pakistan, Taxila, Pakistan \\ Correspondence should be addressed to Qazi Mahmood Ul Hassan; qazimahmood@yahoo.com
}

Received 9 February 2014; Revised 15 April 2014; Accepted 16 April 2014; Published 1 July 2014

Academic Editor: Xiao-Jun Yang

Copyright (C) 2014 Qazi Mahmood Ul Hassan et al. This is an open access article distributed under the Creative Commons Attribution License, which permits unrestricted use, distribution, and reproduction in any medium, provided the original work is properly cited.

\begin{abstract}
We use the fractional derivatives in Caputo's sense to construct exact solutions to fractional fifth order nonlinear evolution equations. A generalized fractional complex transform is appropriately used to convert this equation to ordinary differential equation which subsequently resulted in a number of exact solutions.
\end{abstract}

\section{Introduction}

The concept of differentiation and integration to noninteger order is not new in any case. The notion of fractional calculus emerged when the ideas of classical calculus were proposed by Leibniz, who mentioned it in a letter to L'Hospital in 1695. The foundation of the earliest, more or less, systematic studies can be traced back to the beginning and middle of the 19th century by Liouville in 1832, Riemann in 1853, and Holmgren in 1864, although Euler in 1730, Lagrange in 1772, and others also made contributions. Recently, it has turned out those differential equations involving derivatives of noninteger [1, 2]. For example, the nonlinear oscillation of earthquakes can be modeled with fractional derivatives [3]. There has been some attempt to solve linear problems with multiple fractional derivatives $[3,4]$. Not much work has been done on nonlinear problems and only a few numerical schemes have been proposed for solving nonlinear fractional differential equations $[5,6]$. More recently, applications have included classes of nonlinear equation with multiorder fractional derivatives. The generalized fractional complex transform was applied in [7-13] to convert fractional order differential equation to ordinary differential equation. Finally, by using Exp-function method [14-25] we obtain generalized solitary solutions and periodic solutions. Recently the theory of local fractional integrals and derivatives [26-28] is one of useful tools to handle the fractal and continuously nondifferentiable functions. It is to be tinted that that $c=d$ and $p=q$ are the only relations that can be obtained by applying Exp-function method [29] to any nonlinear ordinary differential equation. Most scientific problems and phenomena in different fields of sciences and engineering occur nonlinearly. Except in a limited number of these problems are linear, this method has been effectively and accurately shown to solve a large class of nonlinear problems. The solution procedure of this method, with the aid of Maple, is of utter simplicity and this method can easily extend to other kinds of nonlinear evolution equations. In engineering and science, scientific phenomena give a variety of solutions that are characterized by distinct features. Traveling waves appear in many distinct physical structures in solitary wave theory [30] such as solitons, kinks, peakons, cuspons, compactons, and many others. Solitons are localized traveling waves which are asymptotically zero at large distances. In other words, solitons are localized wave packets with exponential wings or tails. Solitons are generated from robust balance between nonlinearity and dispersion. Solitons exhibit properties typically associated with particles. Kink waves $[30,31]$ are solitons that rise or descend from one asymptotic state to another and hence another type of traveling waves as in the case of the Burgers 
hierarchy. Peakons, that are peaked solitary wave solutions, are another type of travelling waves as in the case of CamassaHolm equation. For peakons, the traveling wave solutions are smooth except for a peak at a corner of its crest. Peakons are the points at which spatial derivative changes sign so that peakons have a finite jump in 1st derivative of the solution. Cuspons are other forms of solitons where solution exhibits cusps at their crests. Unlike peakons where the derivatives at the peak differ only by a sign, the derivatives at the jump of a cuspon diverge. The compactons are solitons with compact spatial support such that each compacton is a soliton confined to a finite core or a soliton without exponential tails or wings. Other types of travelling waves arise in science such as negatons, positons, and complexitons. In this research, we use the Exp-function method along with generalized fractional complex transform to obtain new Kink waves' solutions for [30-32].

\section{Preliminaries and Notation $[1,2]$}

In this section, we give some basic definitions and properties of the fractional calculus theory $[1,2]$ which will be used further in this work. For the finite derivative in $[a, b]$ we define the following fractional integral and derivatives.

Definition 1. A real function $f(x), x>0$, is said to be in the space $C \mu, \mu \in R$ if there exists a real number $(p>\mu)$ such that $f(x)=x^{p} f_{1}(x)$, where $f_{1}(x)=C(0, \infty)$, and it is said to be in the space $C_{\mu}^{m} \mu$ if $f^{m} \in C \mu, m \in N$.

Definition 2. The Riemann-Liouville fractional integral operator of order $\alpha \geq 0$ of a function $f \in C \mu, \mu \geq-1$, is defined as

$$
\begin{gathered}
J^{\alpha}(x)=\frac{1}{\Gamma(\alpha)} \int_{0}^{x}(x-t)^{\alpha-1} f(t) d t \\
\alpha>0, \quad x>0, \quad J^{0}(x)=f(x) .
\end{gathered}
$$

Properties of the operator $J^{a}$ can be found in [1]; we mention only the following.

For $f \in C \mu, \mu \geq-1, \alpha, \beta \geq 0$, and $\gamma \geq-1$

$$
\begin{gathered}
J^{\alpha} J^{\beta} f(x)=J^{\alpha+B} f(x), \\
J^{\alpha} J^{B} f(x)=J^{B} J^{\alpha} f(x), \\
J^{\alpha} x^{\gamma}=\frac{\Gamma(\gamma+1)}{\Gamma(\alpha+\gamma+1)} x^{\alpha+\gamma},
\end{gathered}
$$

The Riemann-Liouville derivative has certain disadvantages when trying to model real-world phenomena with fractional differential equations. Therefore, we will introduce a modified fractional differential operator proposed by M. Caputo in his work on the theory of viscoelasticity [2].
Definition 3. For $m$ to be the smallest integer that exceeds $\alpha$, the Caputo time fractional derivative operator of order $\alpha>0$ defined as

$$
\begin{aligned}
{ }_{a}^{C} D_{t}^{\alpha} f(t) & =J^{m-\alpha} D^{m} f(t) \\
& =\frac{1}{\Gamma(m-\alpha)} \int_{0}^{t}(t-\tau)^{m-\alpha-1} f^{m}(t) d t
\end{aligned}
$$

for $m-1<\alpha \leq 1 m, m \in N, t>0, f \in C_{-1}^{m}$.

\section{Chain Rule for Fractional Calculus and Fractional Complex Transform}

In [7], the authors used the following chain rule $\partial^{\alpha} u / \partial t^{\alpha}=$ $(\partial u / \partial s)\left(\partial^{\alpha} s / \partial t^{\alpha}\right)$ to convert a fractional differential equation with Jumarie's modification of Riemann-Liouville derivative into its classical differential partner. In [10], the authors showed that this chain rule is invalid and showed following relation $[8]$ :

$$
D_{t}^{a} u=\sigma_{t}^{\prime} \frac{d u}{d \eta} D_{t}^{a} \eta, \quad D_{x}^{a} u=\sigma_{x}^{\prime} \frac{d u}{d \eta} D_{x}^{a} \eta
$$

To determine $\sigma_{s}$, consider a special case as follows:

$$
s=t^{\alpha}, \quad u=s^{m}
$$

and we have

$$
\frac{\partial^{\alpha} u}{\partial t^{\alpha}}=\frac{\Gamma(1+m \alpha) t^{m \alpha-\alpha}}{\Gamma(1+m \alpha-\alpha)}=\sigma \cdot \frac{\partial u}{\partial s}=\sigma m t^{m \alpha-\alpha} .
$$

Thus one can calculate $\sigma_{s}$ as

$$
\sigma_{s}=\frac{\Gamma(1+m \alpha)}{\Gamma(1+m \alpha-\alpha)} .
$$

Other fractional indexes $\left(\sigma_{x}^{\prime}, \sigma_{y}^{\prime}, \sigma_{z}^{\prime}\right)$ can determine in similar way. $\mathrm{Li}$ and $\mathrm{He}$ [3, 7-9] proposed the following fractional complex transform for converting fractional differential equations into ordinary differential equations, so that all analytical methods for advanced calculus can be easily applied to fractional calculus:

$$
u(x, t)=u(\eta), \quad \eta=\frac{k x^{\beta}}{\Gamma(1+\beta)}+\frac{\omega t^{\alpha}}{\Gamma(1+\alpha)}+\frac{M x^{\gamma}}{\Gamma(1+\gamma)}
$$

where $k, \omega$, and $M$ are constants.

\section{Exp-Function Method [33-36]}

Consider the general nonlinear partial differential equation of fractional order:

$$
\begin{array}{r}
P\left(u, u_{t}, u_{x}, u_{x x}, u_{x x x}, \ldots, D_{t}^{\alpha} u, D_{x}^{\alpha} u, D_{x x}^{\alpha} u, \ldots\right)=0, \\
0<\alpha \leq 1,
\end{array}
$$


where $D_{t}^{\alpha} u, D_{x}^{\alpha} u$, and $D_{x x}^{\alpha} u$ are the fractional derivative of $u$ with respect to $t, x, x x$, respectively.

Use

$$
u(x, t)=u(\eta), \quad \eta=\frac{k x^{\beta}}{\Gamma(1+\beta)}+\frac{\omega t^{\alpha}}{\Gamma(1+\alpha)}+\frac{M x^{\gamma}}{\Gamma(1+\gamma)}
$$

where $k, \omega$, and $M$ are constants.

Then (9) becomes

$$
Q\left(u, u^{\prime}, u^{\prime \prime}, u^{\prime \prime \prime}, u^{i v}\right)=0
$$

where the prime denotes derivative with respect to $\eta$. In accordance with Exp-function method, we assume that the wave solution can be expressed in the following form:

$$
u(\eta)=\frac{\sum_{n-c}^{d} a_{n} \exp [n \eta]}{\sum_{m-p}^{q} b_{m} \exp [m \eta]}
$$

where $p, q, c$, and $d$ are positive integers which are known to be further determined and $a_{n}$ and $b_{m}$ are unknown constants. Equation (8) can be rewritten as

$$
u(\eta)=\frac{a_{c} \exp (c \eta)+\cdots+a_{-d} \exp (-d \eta)}{b_{p} \exp (p \eta)+\cdots+b_{-q} \exp (-q \eta)}
$$

This equivalent formulation plays an important and fundamental for finding the analytic solution of problems. $c$ and $p$ can be determined by [29].

\section{Solution Procedure}

Consider the following new fifth order nonlinear $(2+1)$ dimensional evolution equations of fractional order:

$$
D_{t}^{3 \alpha} u-\left(D_{t}^{\alpha} u\right)_{x x x x}-\left(D_{t}^{\alpha} u\right)_{x x}-4\left(u_{x}\left(D_{t}^{\alpha} u\right)_{x}\right)_{x}=0 .
$$

Using (8) in (14) then it can be converted to an ordinary differential equation. Consider

$$
-\omega^{3} \dddot{u}+\omega k^{4} u^{(v)}+k^{2} \omega \ddot{u}+4 \omega k^{3} \dot{u} \ddot{u}=0,
$$

where the prime denotes the derivative with respect to $\eta$. The solution of (15) can be expressed in form (13). To determine the value of $c$ and $p$, by using [26],

$$
p=c, \quad q=d
$$

Case 1. We can freely choose the values of $c$ and $d$, but we will illustrate that the final solution does not strongly depend upon the choice of values of $c$ and $d$. For simplicity, we set $p=c=1$ and $q=d=1$ (15) reduces to

$$
u(\eta)=\frac{a_{1} \exp [\eta]+a_{0}+a_{-1} \exp [-\eta]}{b_{1} \exp [\eta]+a_{0}+b_{-1} \exp [-\eta]}
$$

Substituting (17) into (15), we have

$$
\begin{aligned}
& \frac{1}{A}\left[c_{4} a_{1} \exp [4 \eta]=c_{3} \exp [3 \eta]+c_{2} \exp [2 \eta]+c_{1} \exp [\eta]\right. \\
& +c_{0}+c_{-1} \exp [-\eta]+c_{-2} \exp [-2 \eta] \\
& \left.+c_{-3} \exp [-3 \eta]+c_{-4} \exp [-4 \eta]\right]=0,
\end{aligned}
$$

where $A=\left(b_{1} \exp (\eta)+b_{0}+b_{-1} \exp (-\eta)\right)^{4}$ and $c_{i}$ are constants obtained by Maple software 16. Equating the coefficients of $\exp (n \eta)$ to be zero, we obtain

$$
\begin{gathered}
c_{-4}=0, c_{-3}=0, c_{-2}=0, c_{-1}=0, \\
c_{0}=0, c_{1}=0, c_{2}=0, c_{3}=0, c_{4}=0 .
\end{gathered}
$$

For solution of (19) we have five solution sets satisfying the given (15).

1st Solution Set. Consider

$$
\begin{gathered}
\omega=-\sqrt{k^{2}+1} k, a_{-1}=\frac{b_{-1}\left(3 k b_{0}+a_{0}\right)}{b_{0}}, \\
a_{0}=a_{0}, a_{1}=0, b_{-1}=b_{-1}, b_{0}=b_{0}, b_{1}=0 .
\end{gathered}
$$

We, therefore, obtained the following generalized solitary solution $u(x, t)$ of (14) (Figure 1):

$$
u(x, t)=\frac{b_{-1}\left(3 k b_{0}+a_{0}\right) e^{-k x-\left(\sigma \sqrt{k^{2}+1} k t^{\alpha} / \Gamma(1+\alpha)\right)} / b_{0}+a_{0}}{b_{-1} e^{-k x-\left(\sigma \sqrt{k^{2}+1} k t^{\alpha} / \Gamma(1+\alpha)\right)}+b_{0}} .
$$

2nd Solution Set. Consider

$$
\begin{gathered}
\omega=\sqrt{4 k^{2}+1} k, a_{-1}=\frac{b_{-1}\left(6 k b_{1}+a_{1}\right)}{b_{1}}, \\
a_{0}=a_{0}, a_{1}=a_{1}, b_{-1}=b_{-1}, b_{0}=0, b_{1}=b_{1} .
\end{gathered}
$$

We, therefore, obtained the following generalized solitary solution $u(x, t)$ of (14) (Figure 2):

$$
\begin{gathered}
u(x, t)=\left(b_{-1}\left(6 k b_{1}+a_{1}\right) e^{-k x+\left(\sigma \sqrt{4 k^{2}+1} k t^{\alpha} / \Gamma(1+\alpha)\right)} / b_{1}\right. \\
\left.+a_{1} e^{k x-\left(\sigma \sqrt{4 k^{2}+1} k t^{\alpha} / \Gamma(1+\alpha)\right)}\right) \\
\times\left(b_{-1} e^{-k x+\left(\sigma \sqrt{4 k^{2}+1} k t^{\alpha} / \Gamma(1+\alpha)\right)}\right. \\
\left.+b_{1} e^{k x-\left(\sigma \sqrt{4 k^{2}+1} k t^{\alpha} / \Gamma(1+\alpha)\right)}\right)^{-1} .
\end{gathered}
$$



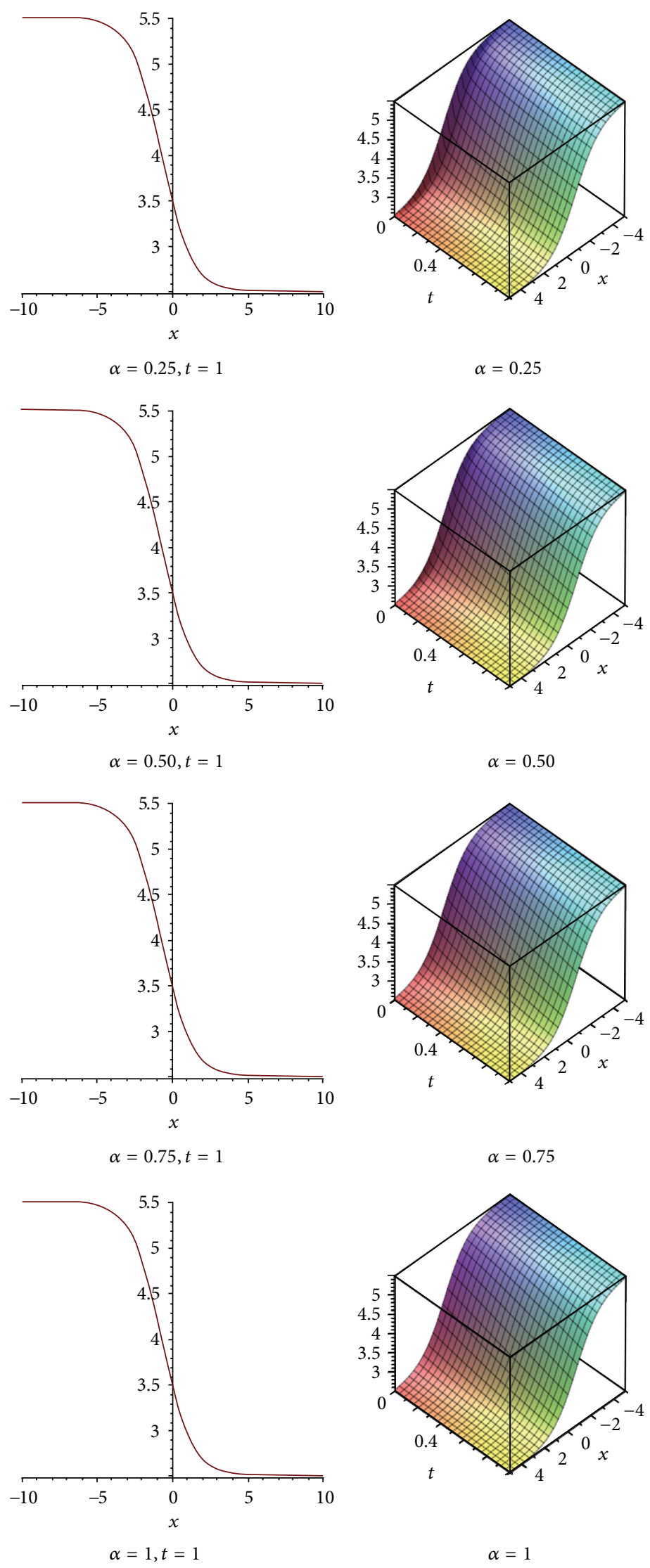

FIGURE 1: Kink waves' solutions of (14) for 1st solution set. 

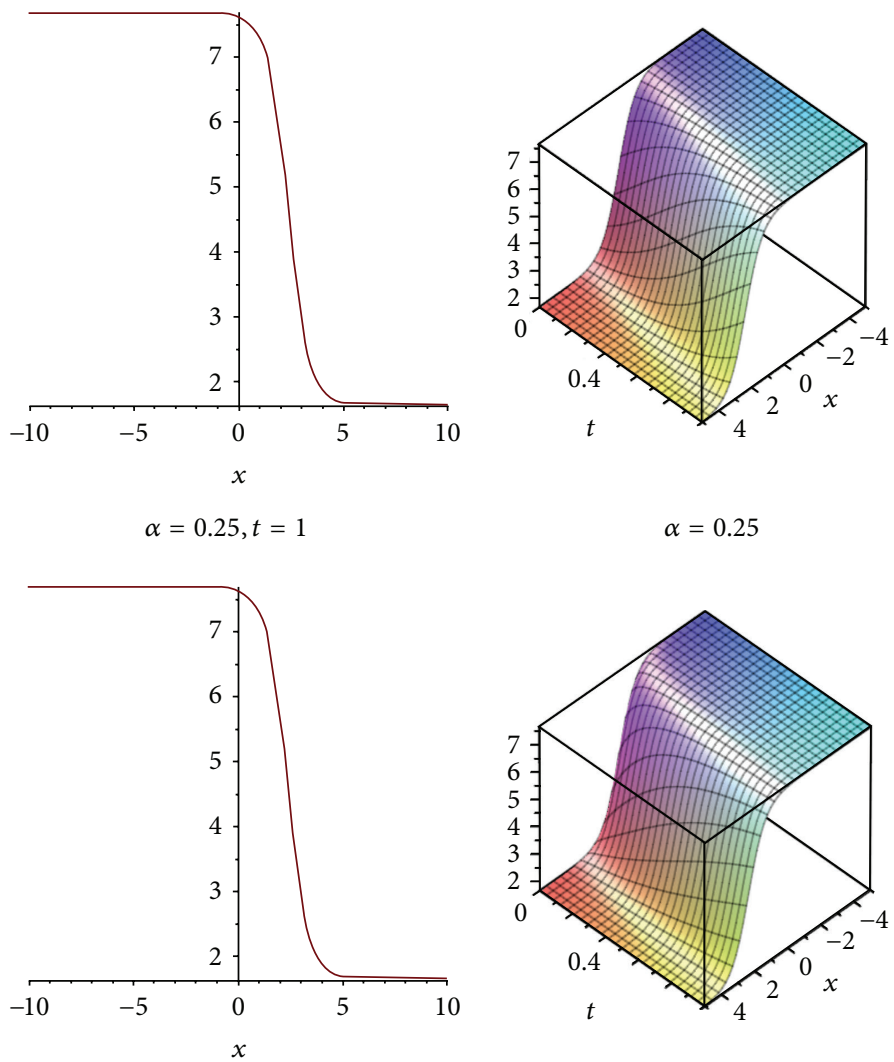

$\alpha=0.50, t=1$
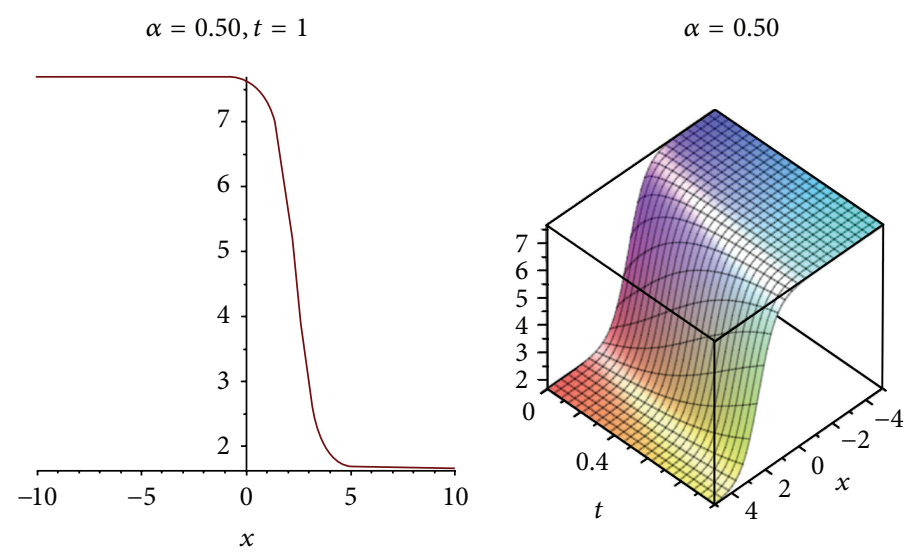

$\alpha=0.75, t=1$

$\alpha=0.75$
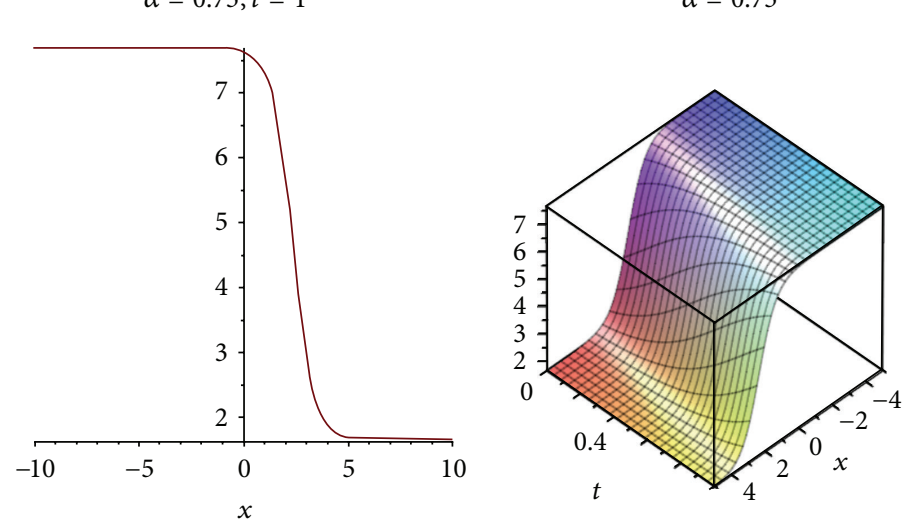

$\alpha=1, t=1$

$\alpha=1$

FIGURE 2: Kink waves' solutions of (14) for 2nd solution set. 
3rd Solution Set. Consider

$$
\begin{gathered}
\omega=\sqrt{k^{2}+1} k, \quad a_{-1}=\frac{b_{-1}\left(3 k b_{0}+a_{0}\right)}{b_{0}}, \\
a_{0}=a_{0}, \quad a_{1}=0, \quad b_{-1}=b_{-1}, \quad b_{0}=b_{0}, \quad b_{1}=0 .
\end{gathered}
$$

We, therefore, obtained the following generalized solitary solution $u(x, t)$ of (14) (Figure 3 ):

$$
u(x, t)=\frac{b_{-1}\left(3 k b_{0}+a_{0}\right) e^{-k x+\left(\sigma \sqrt{k^{2}+1} k t^{\alpha} / \Gamma(1+\alpha)\right)} / b_{0}+a_{0}}{b_{-1} e^{-k x+\left(\sigma \sqrt{k^{2}+1} k t^{\alpha} / \Gamma(1+\alpha)\right)}+b_{0}} .
$$

4th Solution Set. Consider

$$
\begin{gathered}
\omega=\sqrt{k^{2}+1} k \\
a_{-1}=\frac{1}{9} \frac{1}{k^{2} b_{1}^{4}}\left(9 k^{2} a_{0} b_{0} b_{1}^{3}-9 k^{2} a_{1} b_{0}^{2} b_{1}^{2}-3 k a_{0}^{2} b_{1}^{3}+9 k a_{0} a_{1} b_{0} b_{1}^{2}\right. \\
\left.-6 k a_{1}^{2} b_{0}^{2} b_{1}-a_{0}^{2} a_{1} b_{1}^{2}+2 a_{0} a_{1}^{2} b_{0} b_{1}-a_{1}^{3} b_{0}^{2}\right), \\
b_{1}=b_{1}, \quad b_{0}=b_{0}, \\
a_{0}=a_{0}, \quad a_{1}=a_{1}, \\
b_{-1}=\frac{1}{9} \frac{3 k a_{0} b_{0} b_{1}^{2}-3 k a_{1} b_{0}^{2} b_{1}-a_{0}^{2} b_{1}^{2}+2 a_{0} a_{1} b_{0} b_{1}-a_{1}^{2} b_{0}^{2}}{k^{2} b_{1}^{3}} .
\end{gathered}
$$

We, therefore, obtained the following generalized solitary solution $u(x, t)$ of (14) (Figure 4 ):

$$
\begin{aligned}
& u(x, t)=\left(\frac { 1 } { 9 } \frac { 1 } { k ^ { 2 } b _ { 1 } ^ { 4 } } \left(\left(9 k^{2} a_{0} b_{0} b_{1}^{3}-9 k^{2} a_{1} b_{0}^{2} b_{1}^{2}\right.\right.\right. \\
& -3 k a_{0}^{2} b_{1}^{3}+9 k a_{0} a_{1} b_{0} b_{1}^{2} \\
& -6 k a_{1}^{2} b_{0}^{2} b_{1}-a_{0}^{2} a_{1} b_{1}^{2} \\
& \left.+2 a_{0} a_{1}^{2} b_{0} b_{1}-a_{1}^{3} b_{0}^{2}\right) \\
& \left.\times e^{-k x+\left(\sigma \sqrt{k^{2}+1} k t^{\alpha} / \Gamma(1+\alpha)\right)}\right) \\
& \left.+a_{0}+a_{1} e^{k x-\left(\sigma \sqrt{k^{2}+1} k t^{\alpha} / \Gamma(1+\alpha)\right)}\right) \\
& \times\left(\frac { 1 } { 9 } \left(\left(3 k a_{0} b_{0} b_{1}^{2}-3 k a_{1} b_{0}^{2} b_{1}-a_{0}^{2} b_{1}^{2}\right.\right.\right. \\
& \left.+2 a_{0} a_{1} b_{0} b_{1}-a_{1}^{2} b_{0}^{2}\right) \\
& \left.\times e^{-k x+\left(\sigma \sqrt{k^{2}+1} k t^{\alpha} / \Gamma(1+\alpha)\right)}\right)\left(k^{2} b_{1}^{3}\right)^{-1} \\
& \left.+b_{0}+b_{1} e^{k x-\left(\sigma \sqrt{k^{2}+1} k t^{\alpha} / \Gamma(1+\alpha)\right)}\right)^{-1} .
\end{aligned}
$$

5th Solution Set. Consider

$$
\begin{gathered}
\omega=\sqrt{k^{2}+1} k, a_{-1}=\frac{b_{-1}\left(6 k b_{1}+a_{1}\right)}{b_{1}}, \\
a_{0}=0, a_{1}=a_{1}, b_{-1}=b_{-1}, b_{0}=0, b_{1}=b_{1} .
\end{gathered}
$$

We, therefore, obtained the following generalized solitary solution $u(x, t)$ of (14) (Figure 5 ):

$$
u(x, t)=\frac{b_{-1} e^{-k x+\left(\sigma \sqrt{k^{2}+1} k t^{\alpha} / \Gamma(1+\alpha)\right)}+a_{1} e^{k x-\left(\sigma \sqrt{k^{2}+1} k t^{\alpha} / \Gamma(1+\alpha)\right)}}{b_{-1} e^{-k x+\left(\sigma \sqrt{k^{2}+1} k t^{\alpha} / \Gamma(1+\alpha)\right)}+b_{1} e^{k x-\left(\sigma \sqrt{k^{2}+1} k t^{\alpha} / \Gamma(1+\alpha)\right)}} .
$$

Case 2. If $p=c=2$ and $q=d=1$ then trial solution (14) reduces to

$$
u(\eta)=\frac{a_{2} \exp [2 \eta]+a_{1} \exp [\eta]+a_{0}+a_{-1} \exp [-\eta]}{b_{2} \exp [2 \eta]+b_{1} \exp [\eta]+b_{0}+b_{-1} \exp [-\eta]} .
$$

Proceeding as before, we obtain the following.

1st Solution. Consider

$$
\begin{gathered}
a_{-1}=a_{-1}, \quad a_{0}=\frac{a_{-1} b_{0}}{b_{-1}}, \quad a_{1}=\frac{a_{-1} b_{1}}{b_{-1}}, \quad a_{2}=\frac{a_{-1} b_{2}}{b_{-1}}, \\
b_{-1}=b_{-1}, \quad b_{0}=b_{0}, \quad b_{1}=b_{1}, \quad b_{2}=b_{2} .
\end{gathered}
$$

Hence we get the generalized solitary wave solution $u(x, t)$ of (14) (Figure 6):

$$
\begin{array}{r}
u(x, t)=\left(a_{-1} e^{-k x+\left(\sigma \omega k t^{\alpha} / \Gamma(1+\alpha)\right)}+\frac{a_{-1} b_{0}}{b_{-1}}\right. \\
+\frac{a_{-1} b_{0}}{b_{-1}} e^{k x-\left(\sigma \omega k t^{\alpha} / \Gamma(1+\alpha)\right)} \\
\left.+\frac{a_{-1} b_{2}}{b_{-1}} e^{2 k x-2\left(\sigma \omega k t^{\alpha} / \Gamma(1+\alpha)\right)}\right) \\
\times\left(b_{-1} e^{-k x+\left(\sigma \omega k t^{\alpha} / \Gamma(1+\alpha)\right)}+b_{0}\right. \\
\quad+b_{1} e^{k x-\left(\sigma \omega k t^{\alpha} / \Gamma(1+\alpha)\right)} \\
\left.+b_{2} e^{2 k x-2\left(\sigma \omega k t^{\alpha} / \Gamma(1+\alpha)\right)}\right)^{-1} .
\end{array}
$$

In both cases, for different choices of $c, p, d$, and $q$ we get the same soliton solutions which clearly illustrate that final solution does not strongly depend on these parameters.

\section{Conclusions}

Exp-function method is applied to construct solitary solutions of the nonlinear new fifth order evolution equations of fractional orders. The reliability of proposed algorithm is fully supported by the computational work, the subsequent results, and graphical representations. It is observed that Expfunction method is very convenient to apply and is very useful for finding solutions of a wide class of nonlinear problems of fractional orders. 

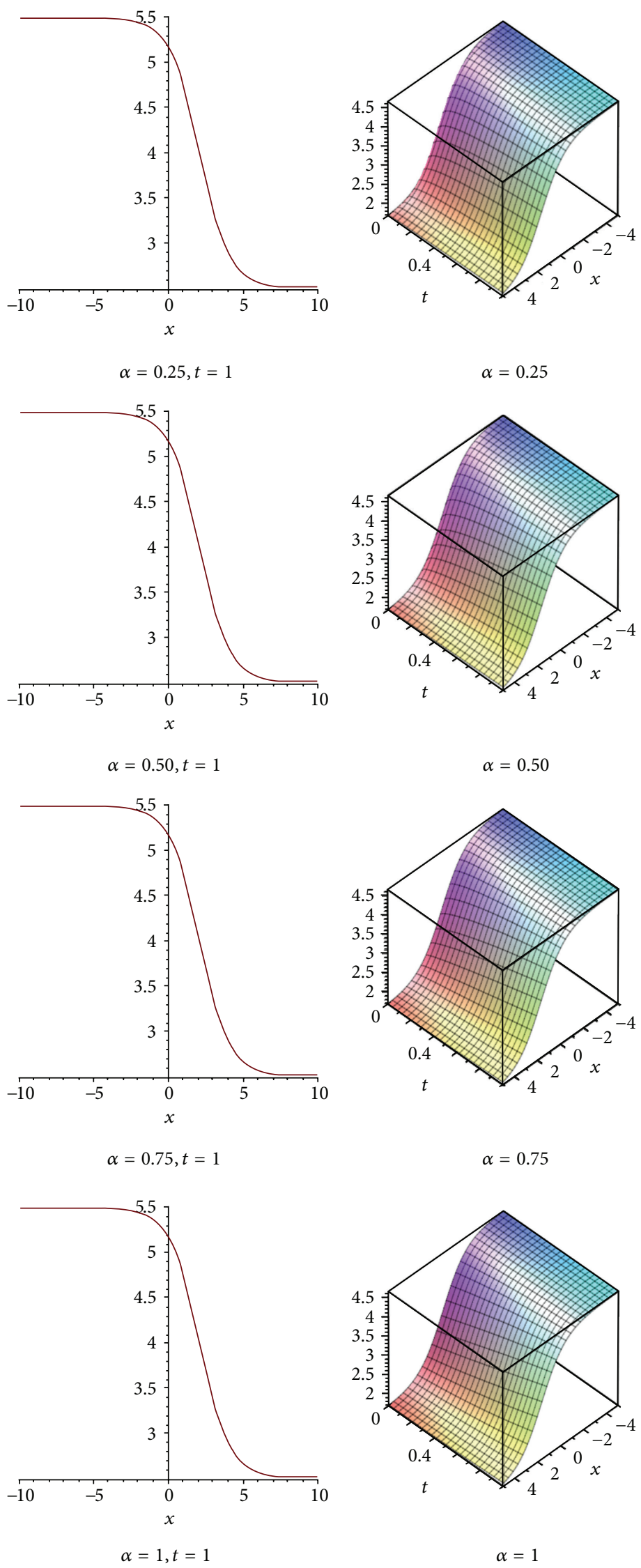

FIgURE 3: Kink waves' solutions of (14) for 3rd solution set. 

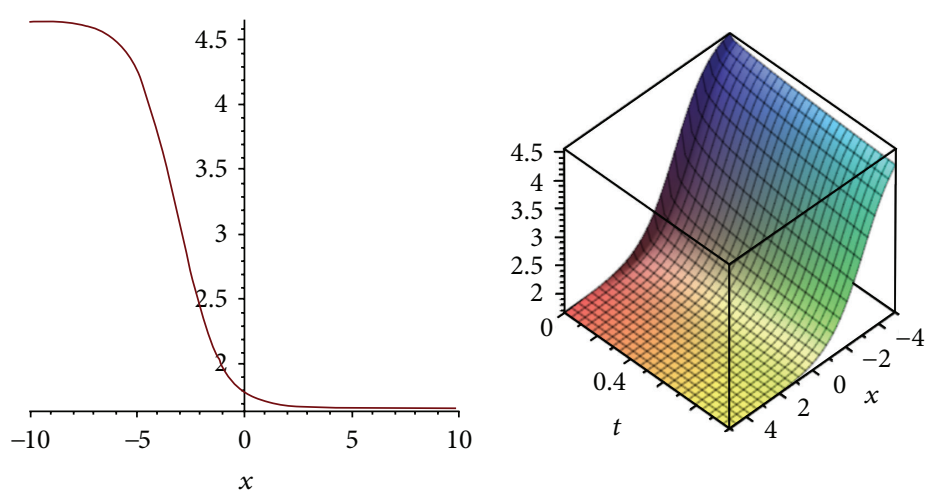

$$
\alpha=0.25, t=1
$$
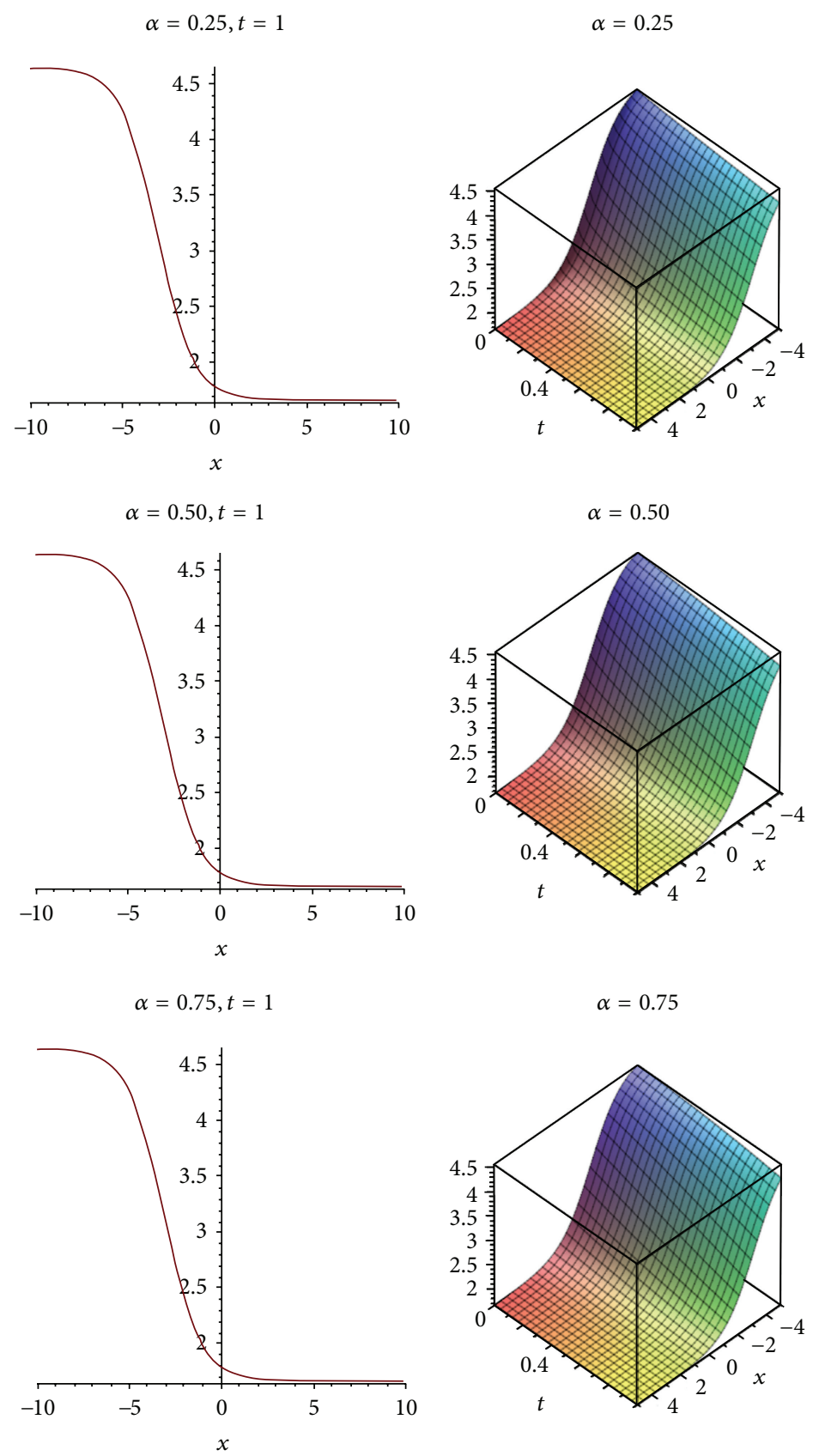

$\alpha=1, t=1$

$\alpha=1$

FigURE 4: Kink waves' solutions of (14) for 4th solution set. 

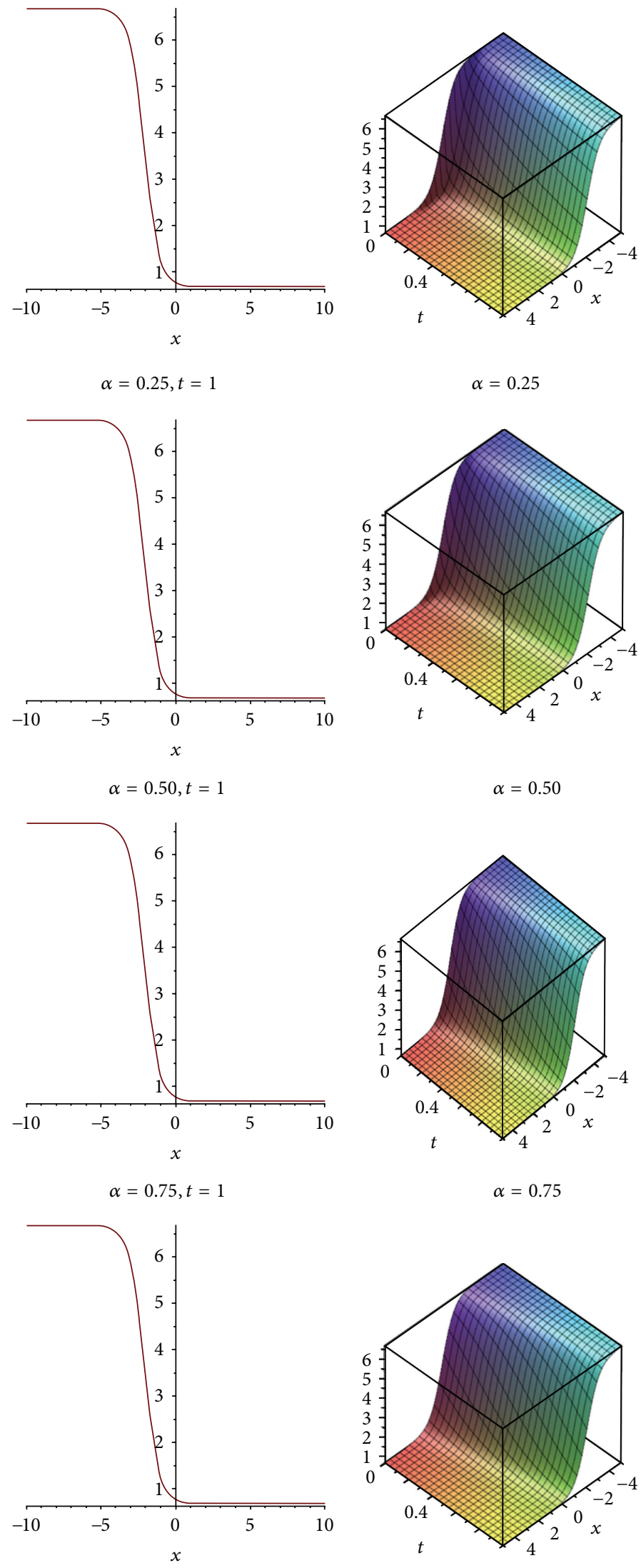

$\alpha=1, t=1$

$\alpha=1$

FIGURE 5: Kink waves' solutions of (14) for 5th solution set. 

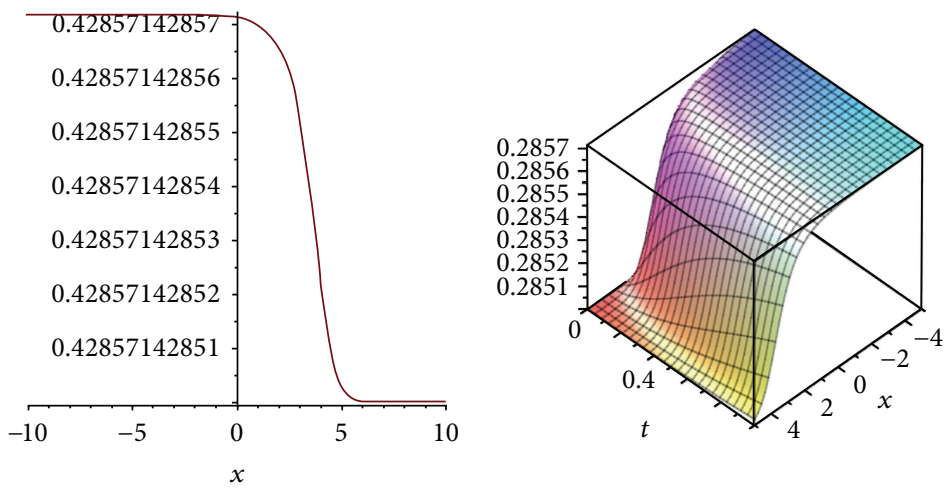

$\alpha=0.25, t=1$

$$
\alpha=0.25
$$
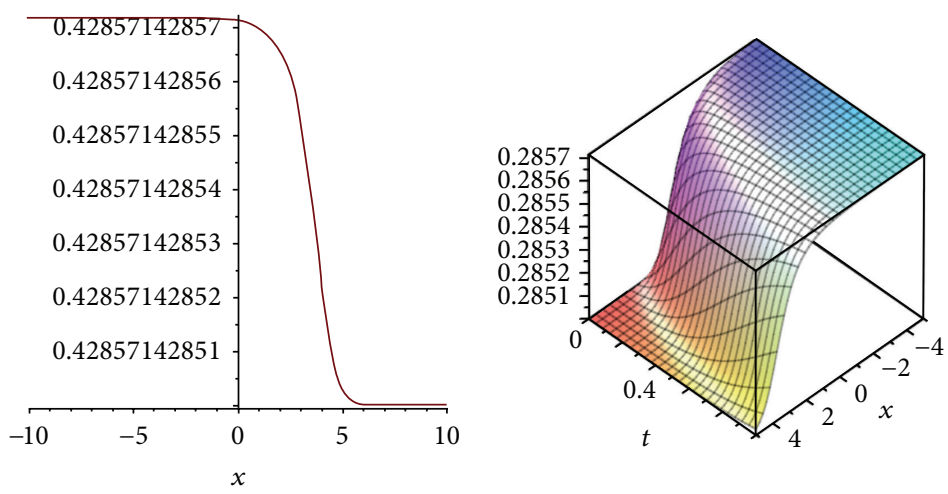

$\alpha=0.50, t=1$
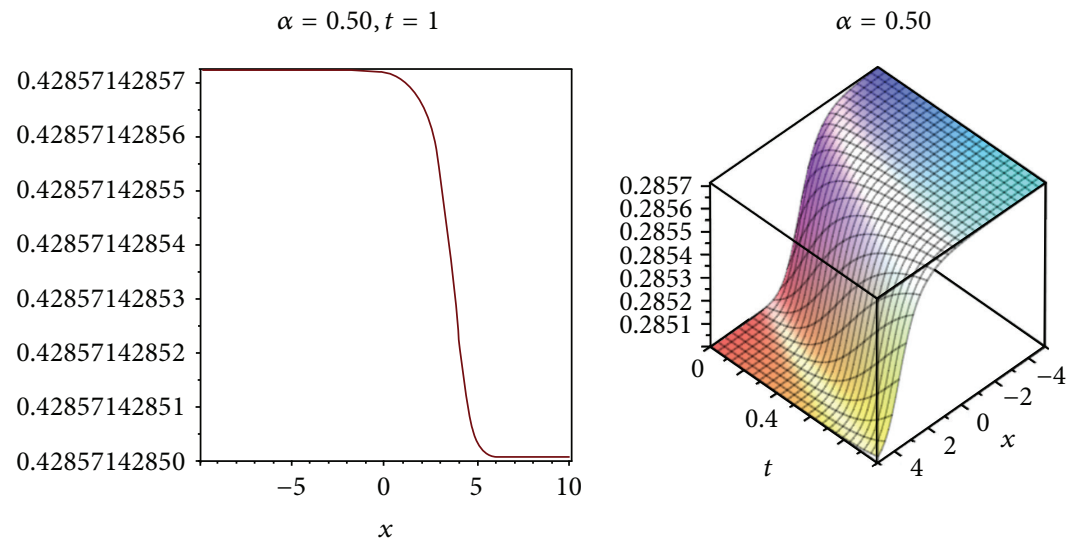

$\alpha=0.75, t=1$
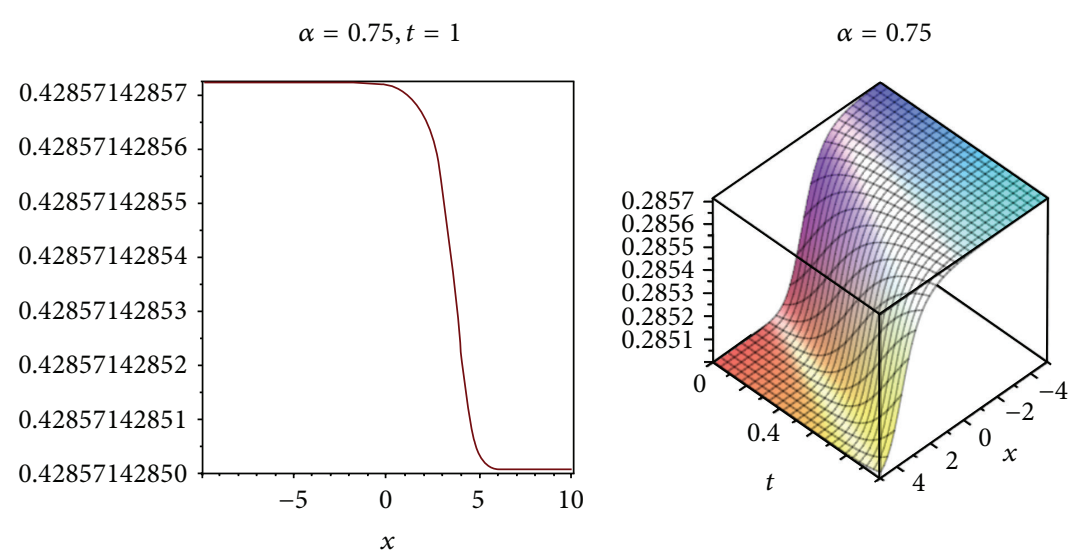

$\alpha=1, t=1$

$\alpha=1$

FIGURE 6: Kink waves' solutions of (14) for 1st solution set of Case 2. 


\section{Conflict of Interests}

The authors declare that there is no conflict of interests regarding the publication of this paper.

\section{References}

[1] A. G. Nikitin and T. A. Barannyk, "Solitary wave and other solutions for nonlinear heat equations," Central European Journal of Mathematics, vol. 2, no. 5, pp. 840-858, 2004.

[2] I. Podlubny, Fractional Differential Equations, vol. 198, Academic Press, San Diego, Calif, USA, 1999.

[3] J. H. He, "Some applications of nonlinear fractional differential equations and their applications," Bulletin of Science, Technology \& Society, vol. 15, no. 2, pp. 86-90, 1999.

[4] K. Diethelm and Y. Luchko, "Numerical solution of linear multi-term initial value problems of fractional order," Journal of Computational Analysis and Applications, vol. 6, no. 3, pp. 243263, 2004.

[5] A. Atangana and N. Bildik, "Existence and numerical solution of the Volterra fractional integral equations of the second kind," Mathematical Problems in Engineering, vol. 2013, Article ID 981526, 11 pages, 2013.

[6] A. Atangana and D. Baleanu, "Numerical solution of a kind of fractional parabolic equations via two difference schemes," Abstract and Applied Analysis, vol. 2013, Article ID 828764, 8 pages, 2013.

[7] Z. B. Li and J. H. He, "Application of the fractional complex transform to fractional differential equations," Nonlinear Science Letters A, vol. 2, no. 3, pp. 121-126, 2011.

[8] Z.-B. Li and J.-H. He, "Fractional complex transform for fractional differential equations," Mathematical \& Computational Applications, vol. 15, no. 5, pp. 970-973, 2010.

[9] J.-H. He, S. K. Elagan, and Z. B. Li, "Geometrical explanation of the fractional complex transform and derivative chain rule for fractional calculus," Physics Letters A, vol. 376, no. 4, pp. 257259, 2012.

[10] R. W. Ibrahim, "Fractional complex transforms for fractional differential equations," Advances in Difference Equations, vol. 2012, article 192, 2012.

[11] J.-H. He, "An elementary introduction to recently developed asymptotic methods and nanomechanics in textile engineering," International Journal of Modern Physics B, vol. 22, no. 21, pp. 3487-3578, 2008.

[12] Z. B. Li and W. H. Zhu, "exact solutions of time-fractional heat conduction equation by the fractional complex transform," Thermal Science, vol. 16, no. 2, pp. 335-338, 2012.

[13] J.-H. He and M. A. Abdou, "New periodic solutions for nonlinear evolution equations using Exp-function method," Chaos, Solitons \& Fractals, vol. 34, no. 5, pp. 1421-1429, 2007.

[14] J.-H. He, "Exp-function method for fractional differential equations," International Journal of Nonlinear Sciences and Numerical Simulation, vol. 14, no. 6, pp. 363-366, 2013.

[15] S. T. Mohyud-Din, M. A. Noor, and K. I. Noor, "Some relatively new techniques for nonlinear problems," Mathematical Problems in Engineering, vol. 2009, Article ID 234849, 25 pages, 2009.

[16] M. A. Noor, S. T. Mohyud-Din, and A. Waheed, "Exp-function method for generalized travelling solutions of master partial differential equations," Acta Applicandae Mathematicae, vol. 104, no. 2, pp. 131-137, 2008.
[17] T. Öziş and C. Köröğlu, "A novel approach for solving the Fisher equation using Exp-function method," Physics Letters A, vol. 372, no. 21, pp. 3836-3840, 2008.

[18] X.-H. Wu and J.-H. He, "EXP-function method and its application to nonlinear equations," Chaos, Solitons \& Fractals, vol. 38, no. 3, pp. 903-910, 2008.

[19] X.-H. Wu and J.-H. He, "Solitary solutions, periodic solutions and compacton-like solutions using the Exp-function method," Computers \& Mathematics with Applications, vol. 54, no. 7-8, pp. 966-986, 2007.

[20] E. Yusufoglu, "New solitonary solutions for the MBBN equations using exp-function method," Physics Letters A, vol. 372, pp. 442-446, 2008.

[21] S. Zhang, "Application of exp-function method to highdimensional nonlinear evolution equation," Chaos, Solitons \& Fractals, vol. 365, pp. 448-455, 2007.

[22] S. D. Zhu, "Exp-function method for the Hybrid-Lattice system, Inter," International Journal of Nonlinear Sciences and Numerical Simulation, vol. 8, pp. 461-464, 2007.

[23] S. D. Zhu, "Exp-function method for the discrete m KdV lattice," International Journal of Nonlinear Sciences and Numerical Simulation, vol. 8, pp. 465-468, 2007.

[24] N. A. Kudryashov, "Exact soliton solutions of a generalized evolution equation of wave dynamics," Journal of Applied Mathematics and Mechanics, vol. 52, no. 3, pp. 465-470, 1988.

[25] S. Momani, "An explicit and numerical solutions of the fractional KdV equation," Mathematics and Computers in Simulation, vol. 70, no. 2, pp. 110-118, 2005.

[26] X. J. Yang, Advanced Local Fractional Calculus and Its Applications, World Science, New York, NY, USA, 2012.

[27] X. J. Yang, Local Fractional Functional Analysis and Its Applications, Asian Academic, Hong Kong, 2011.

[28] X. J. Yang, "Local fractional integral transforms," Progress in Nonlinear Science, vol. 4, pp. 1-225, 2011.

[29] A. Ebaid, "An improvement on the Exp-function method when balancing the highest order linear and nonlinear terms," Journal of Mathematical Analysis and Applications, vol. 392, no. 1, pp. 15, 2012.

[30] A. M. Wazwaz, "New higher-dimensional fifth-order nonlinear equations with multiple soliton solutions," Physica Scripta, vol. 84, Article ID 025007, 2011.

[31] W.-X. Ma, A. Abdeljabbar, and M. G. Asaad, "Wronskian and Grammian solutions to a $(3+1)$-dimensional generalized KP equation," Applied Mathematics and Computation, vol. 217, no. 24, pp. 10016-10023, 2011.

[32] A.-M. Wazwaz, Partial Differential Equations and Solitary Waves Theory, Springer/HEP, Berlin, Germany, 2009.

[33] H. Jafari, N. Kadkhoda, and C. M. Khalique, "Travelling wave solutions of nonlinear evolution equations using the simplest equation method," Computers \& Mathematics with Applications, vol. 64, no. 6, pp. 2084-2088, 2012.

[34] M. A. Abdou, A. A. Soliman, and S. T. Basyony, "New application of Exp-function method for improved Boussinesq equation," Physics Letters A, vol. 369, pp. 469-475, 2007.

[35] J.-H. He and M. A. Abdou, "New periodic solutions for nonlinear evolution equations using Exp-function method," Chaos, Solitons \& Fractals, vol. 34, no. 5, pp. 1421-1429, 2007.

[36] M. A. Noor, S. T. Mohyud-Din, and A. Waheed, "Exp-function method for solving Kuramoto-Sivashinsky and Boussinesq equations," Journal of Applied Mathematics and Computing, vol. 29, no. 1-2, pp. 1-13, 2009. 


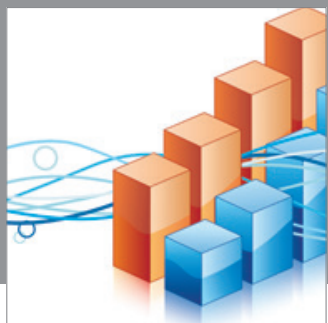

Advances in

Operations Research

mansans

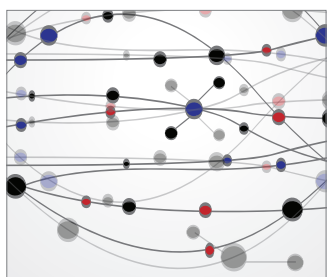

The Scientific World Journal
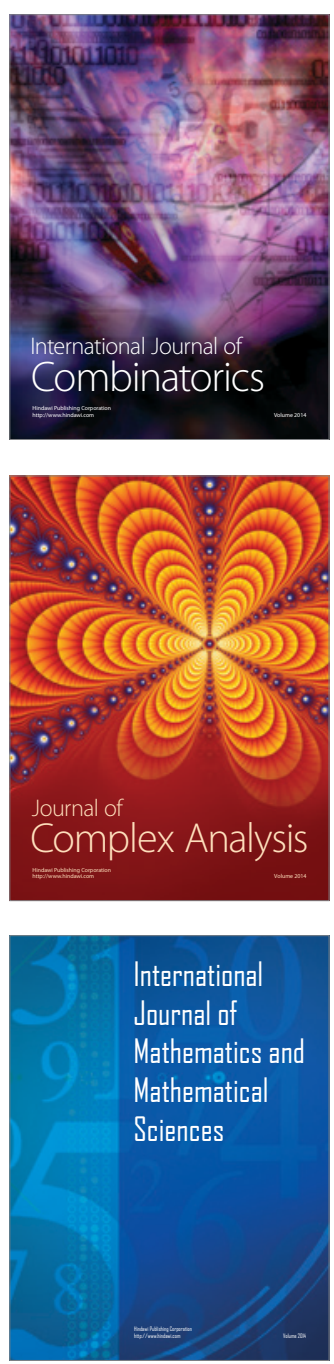
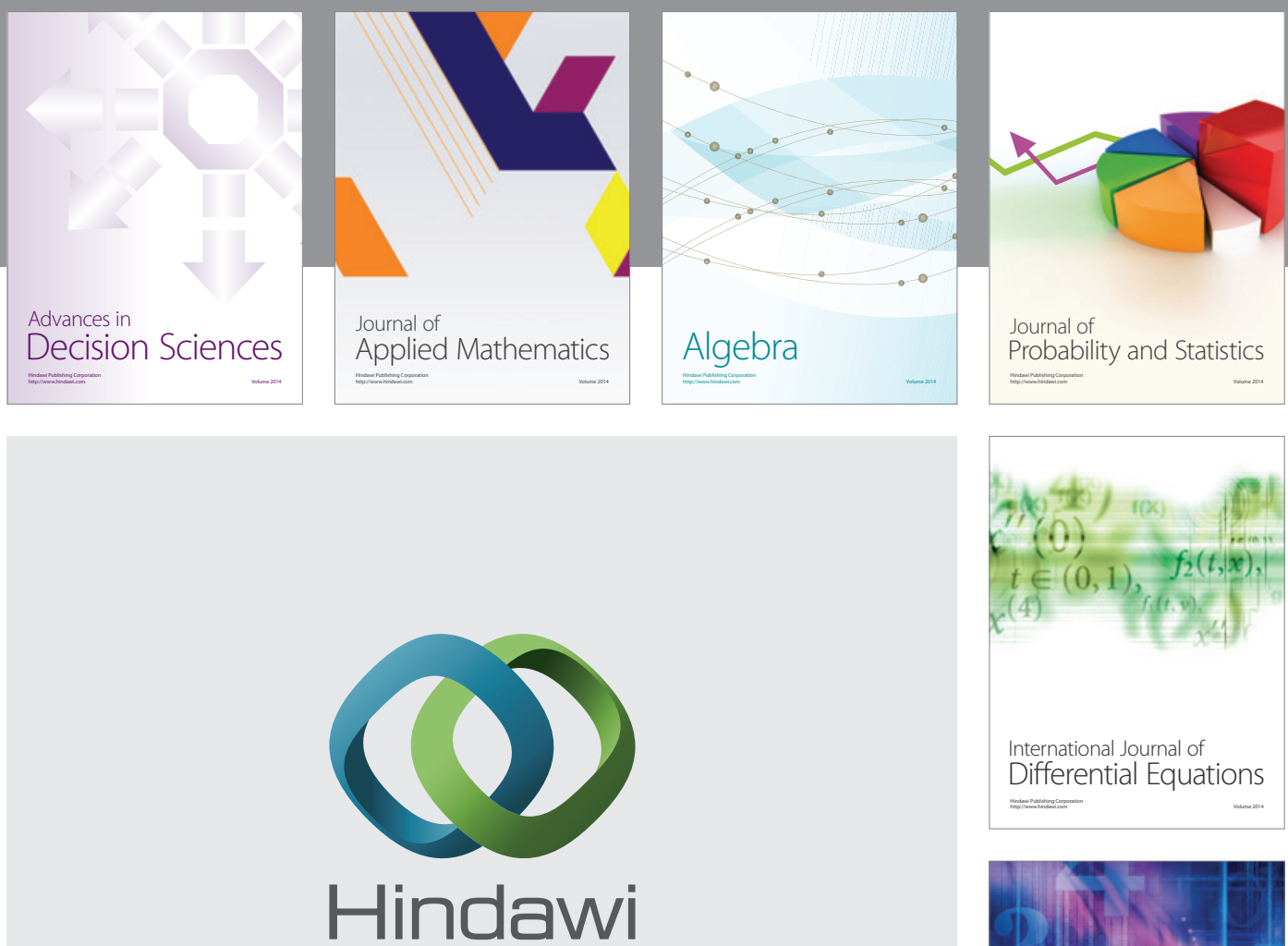

Submit your manuscripts at http://www.hindawi.com
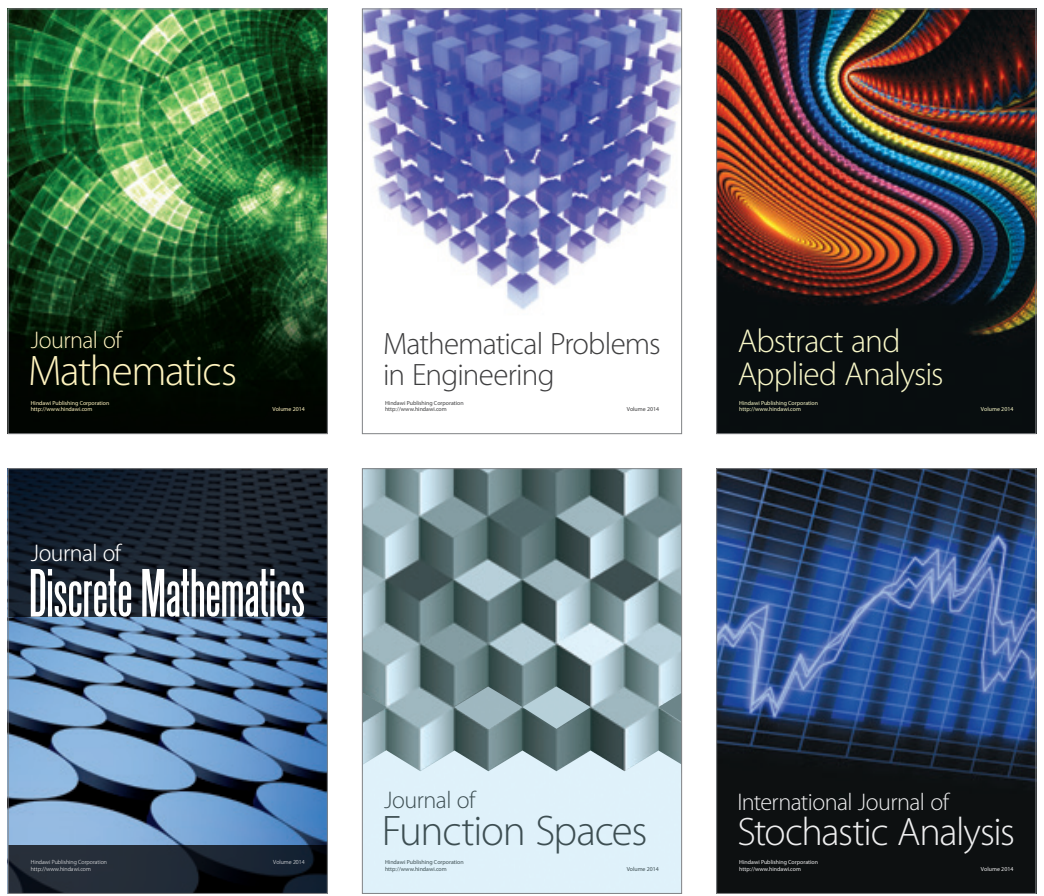

Journal of

Function Spaces

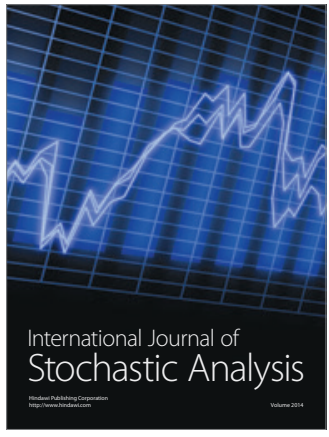

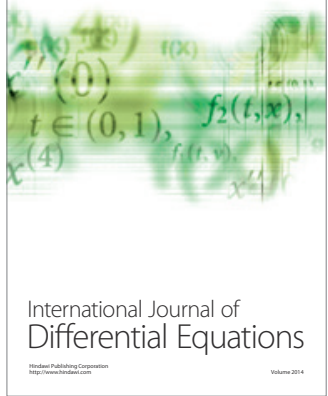
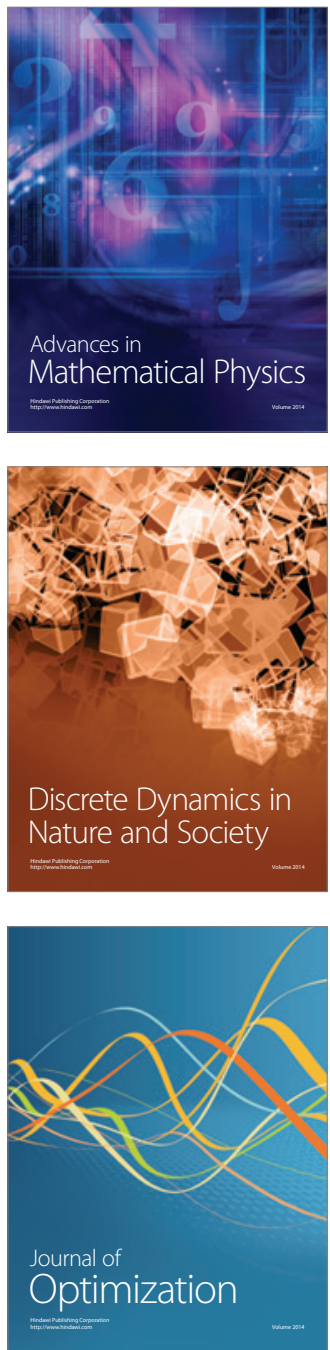